\title{
Sigmoid kinetics of protein crystal nucleation
}

\author{
Christo N. Nanev*, Vesselin D. Tonchev \\ Rostislaw Kaischew Institute of Physical Chemistry, Bulgarian Academy of Sciences, \\ 1113 Sofia, Bulgaria
}

\begin{abstract}
A non-linear differential equation expressing the new phase nucleation rate in the different steps of the process (non-stationary and stationary nucleation and in the plateau region) is derived from basic principles of the nucleation theory. It is shown that one and the same sigmoid (logistic) function describes both nucleation scenarios: the one according to the classical theory, and the other according to the modern two-stage mechanism of protein crystal formation. Comparison to experimental data on both insulin crystal nucleation kinetics and on bovine $\beta$-lactoglobulin crystallization indicates a good agreement with the sigmoidal prediction. Experimental data for electrochemical nucleation and glass crystallization obey the same sigmoid time dependence, and suggest universality of this nucleation kinetics law.
\end{abstract}

Key words: A1. Nucleation; A1. Protein crystal nucleation kinetics; A1. Self-limiting nucleation rate increase; A1. Separation of crystal nucleation and growth; A1.Universality of sigmoid nucleation kinetics law.

* Corresponding Author: nanev@ipc.bas.bg; Phone: (359-2) 8566458; Fax: (359-2) 971 2688. 


\section{Introduction}

One of nucleation theory's main objectives is to provide expressions for the nucleation rate, $\mathrm{d} n / \mathrm{d} t$, which is the number of the new phase nuclei, $n$, appearing in unit volume (1 $\mathrm{cm}^{3}$ ) per unit time, $t=1 \mathrm{sec}$. Since nucleation rate cannot be measured directly, plots of experimentally determined number densities of nuclei formed per unit time are used. Such determinations are done by separating nucleation and growth stages (see §3.1).

Experimental studies under constant supersaturation, e.g. [1-2] have indicated that after some initial time-lag, the early crystal nucleation stage is slow, then speeds up gradually (nearly exponential upside), and slows down by approaching the final stage where saturation is established, i.e. a nucleation rate of zero. Many processes in nature (e.g. plant growth), human society and diverse inorganic and bio-systems, including amyloid fibril formation [3] run in a similar way. Such S-shaped progressions that have slow beginnings, then accelerate and over time reach a climax are described quantitatively by sigmoid (logistic) functions [4-6].

It is the aim of this paper to elucidate theoretically the increase in number densities of new phase nuclei, $n$, formed under constant supersaturation during nucleation time, $t$. It is worth noting that when nucleation is effectively arrested, e.g. using the classical nucleation-growth-separation principle, NGSP (in which the nucleation time is set short, and the growth of the nuclei during this stage is negligible, §3.1), the consideration is much simpler than in Kolmogorov-Johnson-Mehl-Avrami theory [7-11].

\section{Theoretical}

According to the nucleation theory, when supersaturation is established in a system, cluster size distribution changes first from the previous equilibrium distribution to a new distribution corresponding to the metastable state; this is the physical reason for the so-called induction time or non-stationary time lag in nucleation. Due to the random density fluctuations in the mother phase, the larger the cluster, the longer it takes for it to emerge, and hence, the new distribution of clusters, smaller than the critical size has to be formed before the first critical nucleus appears; it is the latest to appear. However, the gradual accommodation to the cluster size distribution corresponding to the metastable state condition continues even after the first nucleus appears, i.e. throughout the entire 
initial non-steady-state nucleation period. Thus, the number of nuclei precursors' increases with time, and augments the basis for (accelerated) nucleation. It is worth noting that according to the classical nucleation theory (and to Szilard chain) critically sized clusters form through attachment of one molecule to subcritical clusters of size $\left(i^{*}\right.$ $1), i^{*}$ being the number of molecules constituting the critical cluster. However, according to Frenkel's “chemical” approach (and some more recent ideas), monomer attachment may not be the sole nucleation scenario; critical and/or supercritical nuclei can form by cluster coalescence events as well. Somehow or other, the nucleation rate $\mathrm{d} n / \mathrm{d} t$ initiates proportionally to the critical nuclei number density, $n$, multiplied by the "birth" frequency, $k: \mathrm{d} n / \mathrm{d} t=k n$. Thus, starting with a single nucleus, the nucleation process advances with time in an exponential manner: $n=\exp (k t)$.

The initial non-steady-state period is followed by a stationary nucleation, lasting for a limited time only; afterwards, $\mathrm{d} n / \mathrm{d} t$ decreases continuously, until becoming zero in the plateau region of the $n$ vs. $t$ dependences. This is a typical example of a self-limiting increase where deceleration can be attributed to exhaustion of particles (and/or centers), which are active for nucleation; known generally as nucleants, such particles are always present in protein solutions. The nucleants are gradually occupied by nuclei (generated by rate $\mathrm{kn}$ ) and ingested by local nucleation exclusion zones, which form around growing nuclei by rate $\omega n$. Because the two decelerating factors act in parallel, the probability for their simultaneous action is $\omega k n^{2}, \omega$ being a constant: $\mathrm{d} n / \mathrm{d} t=k n-\omega k n^{2}$. The condition to have a plateau in the $n$ vs. $t$ dependence is $\mathrm{d} n / \mathrm{d} t=0$. Hence, $\omega=1 / n_{\mathrm{s}}$, where $n_{\mathrm{s}}$ is the saturated nuclei number density, and

$$
\frac{d n}{d t}=k n\left(1-\frac{n}{n_{s}}\right)
$$

This is a first-order non-linear differential equation that can be solved exactly. The integral of this equation shows a sigmoidal dependence of $n$ on $t$, Fig. 1. Note however that the overall supersaturation does not change with time for short nucleation times only (e.g. as by using NGSP); the reason is the negligible amount of solute, which is included in the extremely small nuclei.

Nucleation acceleration and deceleration tendencies have to equilibrate. This occurs at the point when the maximum nucleation rate is reached. Hence, at this point the 
second derivative is $\mathrm{d}^{2} n / \mathrm{d} t^{2}=0$, and $n / n_{\mathrm{s}}=0.5$. Putting this value in eq. (1), we obtain the maximum nucleation rate

$$
(\mathrm{d} n / \mathrm{d} t)_{\max }=k n_{\mathrm{s}} / 4
$$

It is seen that $k$ models the maximum nucleation rate.

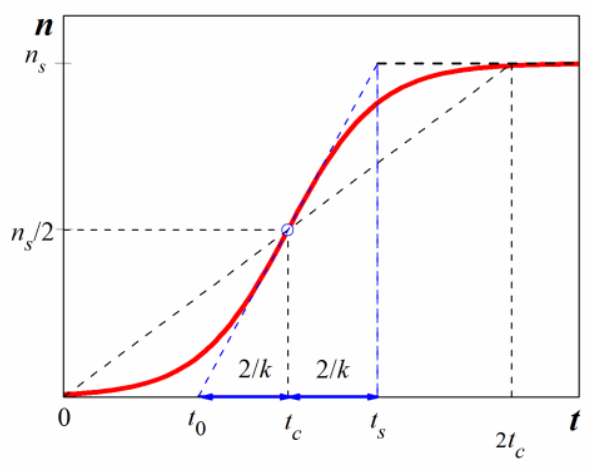

Fig. 1 Sigmoid curve with characteristic points (see text); the nucleation induction time lies in the negative part of abscissa.

Equation eq. (2) is used to calculate the time $t_{\mathrm{c}}$, when the maximum nucleation rate is reached, and $n=n_{\mathrm{s}} / 2$. With this end in view, we draw a straight line, which is tangential to the sigmoid point $n=n_{\mathrm{S}} / 2$ (the dashed line in Fig. 1); its linear equation is

$n=a+k n_{\mathrm{s}} t / 4$

For $t=t_{\mathrm{c}}$ at $n=n_{\mathrm{s}} / 2$, it results in

$a=n_{\mathrm{s}}\left(1-k t_{\mathrm{c}} / 2\right) / 2$

And with this $a$-value the tangential line equation transforms in

$$
n=\frac{n_{s}}{2}\left(1-\frac{k t_{c}}{2}\right)+\frac{k n_{s}}{4} t
$$

Now, denoting the intersection point of the tangential straight line with the abscissa by $t_{\mathrm{o}}$ (Fig. 1), we obtain

$$
t_{0}=t_{c}-\frac{2}{k}, \text { and } t_{\mathrm{c}}=t_{\mathrm{o}}+2 / k
$$

It should be noted that $t_{\mathrm{o}}$ is related to the induction time $\tau$ (see $\S 3.3$ ).

The symmetry of the sigmoid curve shows that $n_{\mathrm{s}}$ is reached at time $t_{\mathrm{p}}=2 t_{\mathrm{c}}$ (Fig. 1). With the above parameters, and after some mathematical transformations, the integral of eq. (1) results in the following logistic (sigmoid) function 


$$
n=\frac{n_{s}}{1+\exp \left[-k\left(t-t_{c}\right)\right]}
$$

Sigmoid's midpoint is reached at $t=t_{\mathrm{c}}$, because $\exp \left[-k\left(t-t_{\mathrm{c}}\right)\right]=1$; and when $\exp [k(t-$ $\left.\left.t_{\mathrm{c}}\right)\right] \gg 1, n \rightarrow n_{\mathrm{s}}$, which is the final nucleation stage.

The coefficients $k$ is the rate determining constant (it has dimensions of reciprocal time), and is of special interest in the physics of new phase nucleation [12]. Equation (1) shows that at the beginning of the nucleation process, when $n=1, \mathrm{~d} n / \mathrm{d} t=k\left(n_{\mathrm{s}}-1\right) / n_{\mathrm{s}}$; and with $n_{\mathrm{s}} \gg 1, \mathrm{~d} n / \mathrm{d} t \approx k$. However, there are more practical ways to determine $k$. Firstly, by fitting experimental data in eq. (6) the independent parameters $k$ and $t_{\mathrm{c}}$ are obtained, e.g. Table 1 (and the comments in \$3.2). Still another possibility to evaluate $k$ is by using the time $t_{\mathrm{s}}$, when the saturated nuclei number density $n_{\mathrm{s}}$ is reached (see Fig. 1); and according to the tangential line equation (3.1)

$$
t_{\mathrm{s}}=t_{\mathrm{c}}+2 / k
$$

which combined with eq. (5) renders (also see Fig. 1)

$$
k=4 /\left(t_{\mathrm{s}}-t_{\mathrm{o}}\right)
$$

Besides the simultaneous action of the two decelerating factors, there are two more possibilities to consider. The first one assumes an exceptionally pure system (e.g. vapor phase) where no nucleants are present and the homogeneous nucleation is decelerated solely because of the nucleation excluded zones arising around the nuclei; increasing in number, such zones may decrease noticeably the volume where nucleation can still proceed; an exponential increase in nuclei number density is expected to occur at constant supersaturation. But system's overall supersaturation is constant for sufficiently short nucleation time only; soon or latter, it drops due to the growth of the crystals. Thus, the nucleation process decelerates, and again sigmoid time dependence should result.

The second possibility refers to the case when nucleation is decelerated only due to the exhaustion of the present active crystal nucleation particles/centers. This topic had been discussed by many authors, e.g. Robins and Rhodin [13], Kaischew and Mutaftschiew [14], just to mention some (an extensive list of such authors is presented in Chapter 32 by Kashchiev [15]). Recently, Milchev [16] has shown that active centers can appear on the electrode surface during the course of the nucleation process itself (which poses some complications in studying electrochemical nucleation). 


\section{Experimental}

\subsection{Study of insulin crystal nucleation; nucleation-growth-separation principle}

Nucleation of rhombohedral insulin crystals (Fig. 2) was studied experimentally [1]. The investigation was performed according to a modernized variant of NGSP (originally devised by Tammann [17]), which although very simple, provides a unique possibility for a direct nucleation study - without ever actually seeing the nuclei themselves: Initially, one rapidly supersaturates the system so that nucleation occurs. To avoid the growth of the arising nuclei however, the nucleation period is set short. The NGSP was devised due to the inherent impossibility to observe elementary acts of crystal nucleation, which proceed at a molecular scale. Therefore, to make nucleated crystallites visible under light microscope the nucleation process is abruptly stopped and the created nuclei are let to grow. This is achieved by rapidly lowering the supersaturation below the upper limit of the so-called metastable zone, i.e. below the threshold limiting the crystal nucleation process. Depriving the system of its capability to further produce nuclei, only the existing super-critically sized clusters are let grow to microscopically visible crystals (so, they are "developed"). Counting them and plotting crystal number density $n$ vs the nucleation time $t$, the stationary nucleation rate for the corresponding supersaturation is determined from the linear parts of the $n$ vs. $t$ curves. This is the conventional way for determining the stationary nucleation rate [1]. The NGSP was used for studying protein crystal nucleation in the groups of Vekilov and Veesler with lysozyme [18-21] and bovine pancreatic trypsin inhibitor [22] (which has a retrograde temperature dependent solubility).

Custom made quasi-2D all-glass cells [23] were applied in our investigation; their volume is $0.35 \mathrm{~cm}^{3}$. Crystal nucleation is a random process. To reduce the inherent data scatter we count all crystals in the entire cell, and average the results. The procedure is simple, but reliable: A grid is drawn on the cell by means of differently colored (waterresistant) felt-tip pens. In this way the meshes are distinguished clearly, which avoids uncertainty in crystal counting; the latter being done using CCD-camera photo images. Because cell area is relatively large on a micro-scale (about $7 \mathrm{~cm}^{2}$ ), several CCD-images are needed; their number depends on the microscopic magnification. Counting the crystals on those images, we observe that crystal number varies from mesh to mesh 
significantly. Therefore, additional experiments are devoted to this point. Increasing the temperature above $45^{\circ} \mathrm{C}$ we initially dissolved all insulin crystals in the cell. Then, decreasing the temperature of the same solution we grew new crystals. In doing so, we observe that the new born crystals appear on new places in the cell; even on the same cell place crystal number density and distribution change with every try. The conclusion is evident, only the mean nuclei number densities, averaged over the entire cell, provide useful data.

Heterogeneous (on-glass) crystal nucleation was differentiated from one in bulk by focusing the microscope on the upper glass plate of the cell. Insulin crystal nucleation at the air/solution interface and in the three-phase angle air/solution/glass was studied during the same experiment on appropriately created air bubbles in the cells. It turned out that the crystal nucleation in bulk solution is highly prevailing; we supposed that the nuclei were formed on some foreign particles of biological origin [1]; such particles are always present in any protein solution.
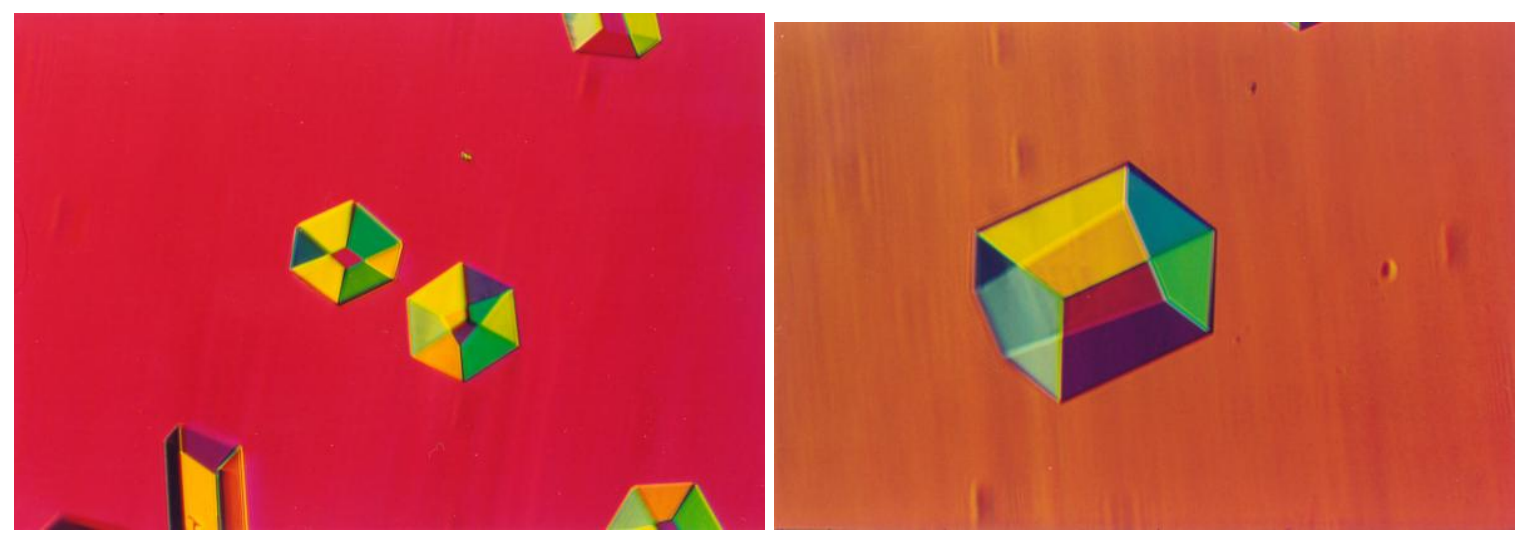

Fig. 2 Rhombohedral insulin crystals, about $0.5 \mathrm{~mm}$ in size; interference contrast by differential image splitting.

Ten identical quasi-2D-cells have been loaded with the same insulin solution, and subsequently were immersed in a water bath at $4^{\circ} \mathrm{C}$; insulin solubility is temperature dependent, and thus, temperature was used as an instrument to produce insulin crystal nuclei. After the desired nucleation time elapsed for each individual cell, consecutively, one by one the cells were transferred in another water bath having appropriate growth 
temperature, which was selected in the temperature interval where nuclei number density is temperature independent [24].

Insulin from porcine pancreas (BioChemika, $\geq 85 \%$ (GE), $\sim 24 \mathrm{IU} / \mathrm{mg}$ ) was crystallized without further purification. The crystallization conditions were: $0.001 \mathrm{M}$ $\mathrm{HCl}$ and $0.005 \mathrm{M} \mathrm{ZnCl}_{2}, \mathrm{pH}=6.98$, by $0.05 \mathrm{M}$ trisodium citrate, $15 \%(\mathrm{v} / \mathrm{v})$ acetone. Initially, insulin was dissolved in highly diluted $\mathrm{HCl}$. Then consecutively citrate buffer, $\mathrm{ZnCl}_{2}$, and finally acetone were added. The solution and all quasi-2D glass cells were tempered to $50^{\circ} \mathrm{C}$. Subsequently, the temperature of the cells and the protein solution was decreased to $30^{\circ} \mathrm{C}$, which ensures the starting metastable condition of the solution introduced in the cells (for further details see [1]).

\subsection{Insulin crystal nucleation kinetics obeys sigmoid law}

Figure $3 \mathrm{a}$ shows the S-shaped plot, according to eq. (6), for insulin crystal nucleation at dimensionless supersaturation 2.99. To probe logistic functional dependence generality, experimental data on insulin crystal nucleation measured at 7 different supersaturations [1] were plotted according to eq. (6). Data for all 4 cases (nucleation in bulk solution, on-glass surface, at the air/solution interface and in the three-phase angle air/solution/glass) fitted well to this function. As an example, Figure $3 b$ shows the plot for bulk solution where nucleation is highly prevailing. Figure $3 \mathrm{c}$ exhibits that all points for $n / n_{\mathrm{s}}$ vs. $t / t_{\mathrm{p}}$ curves less than 0.5 are situated below the dashed straight line (with coordinates (00), (11)), while the points for greater (up to 1) $n / n_{\mathrm{s}}$ vs. $t / t_{\mathrm{p}}$ values are positioned above this line. This proves the S-shape of the curves.

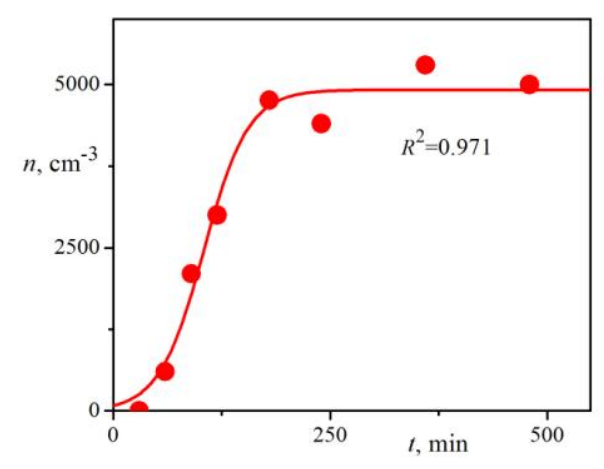

Fig. 3a

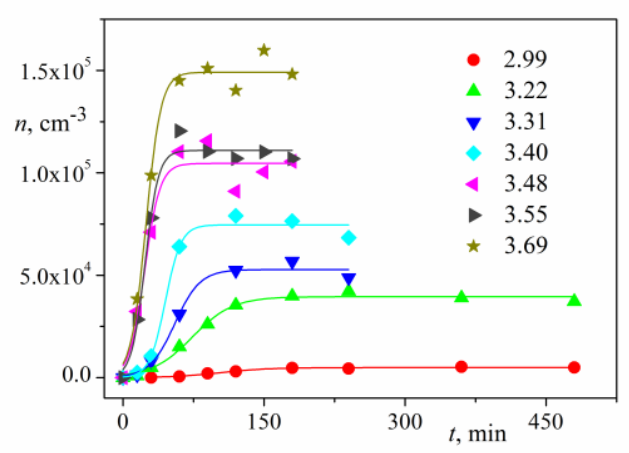

Fig. 3b 


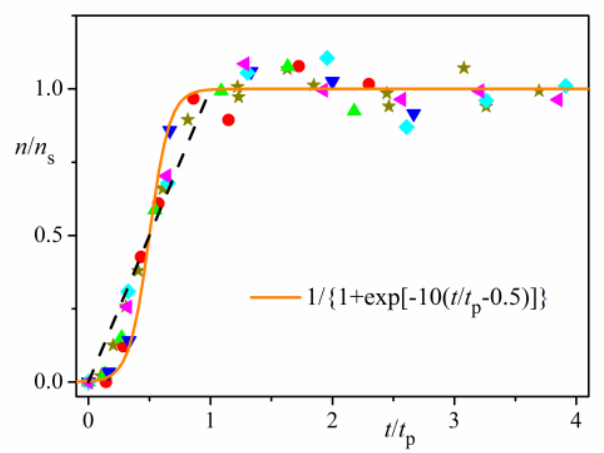

Fig. 3c

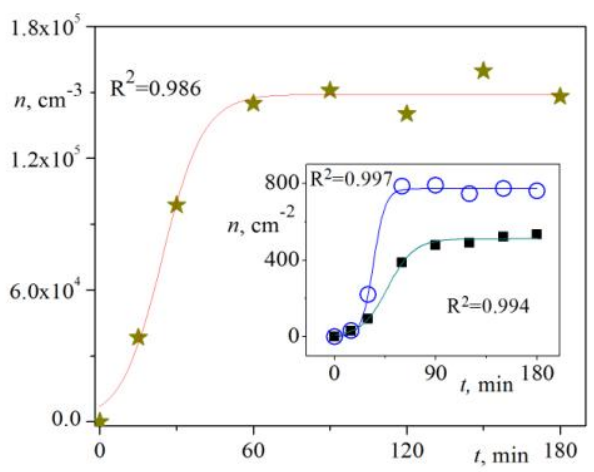

Fig. 4

Fig. 3a Plot of $n$ vs. $t$ data for insulin crystal nucleation in bulk solution at dimensionless supersaturation 2.99; the sizes of the marks reflect the inherent data scatter.

Fig. 3b Experimental data, $n$ vs. $t$, are plotted according to eq. (6) for bulk insulin crystal nucleation; the numbers on the right-hand side show dimensionless supersaturations, $\ln \left(c / c_{\mathrm{e}}\right)$, where $c$ is the actual insulin concentration, $c_{\mathrm{e}}$ being the equilibrium one.

Fig. 3c Logistic functional dependences of $n / n_{\mathrm{s}}$ vs. $t / t_{\mathrm{p}}$ for bulk insulin crystal nucleation (proceeding at the 7 fixed supersaturations shown in Fig. 3b) plotted according to eq. (6); the orange curve is drawn with $k t_{\mathrm{p}} \approx 10$.

Fig. 4 Plots of $n$ vs. $t$, measured for insulin crystal nucleation at supersaturation 3.69: stars represent data for nucleation in bulk solution, $n\left[\mathrm{~cm}^{-3}\right]$; the inserted graph (dimensions of $n\left[\mathrm{~cm}^{-2}\right]$ ) shows nucleation on glass surface (open circles) and nucleation on the air/solution interface (black squares).

The generality of the logistic function dependence is confirmed by plots of data for insulin crystal nucleation in bulk solution, on glass surface and on the air/solution interface, at the same dimensionless supersaturation 3.69, Fig. 4. The good quality of the fits in Fig. 3a and Fig. 4 is seen from the $\mathrm{R}^{2}$-values (for the remaining curves see Table 1). Simultaneously, the good fit of the experimental data in Fig. 3c proves the role that foreign particles of biological origin play in the nucleation process. Further to that, the values of bulk insulin crystal nucleation rates obtained from the logistic dependences are closely similar to those in [1]; and since logarithms of the nucleation rates are used in the 
calculation of the nucleation energy barrier and the numbers of insulin molecules constituting the critical nuclei, the results are the same as those calculated earlier [1].

Table 1 Data calculated using eq. (6) for insulin crystal nucleation in bulk solution, on glass surface, at the air/solution interface and in the three-phase angle air/solution/glass.

\begin{tabular}{||l|l|l|l|l||c||c|c||}
\hline \multirow{2}{*}{$\ln \left(c / c_{\mathrm{e}}\right)$} & \multicolumn{4}{|c||}{ bulk insulin crystal nucleation } & on-glass & air/solution & $\begin{array}{c}\text { 3-phase } \\
\text { angle }\end{array}$ \\
\cline { 2 - 9 } & $n_{\mathrm{s}}$ & $t_{\mathrm{c}}[\mathrm{s}]$ & $\begin{array}{c}k \times 10^{4} \\
{\left[\mathrm{~s}^{-1}\right]}\end{array}$ & $R^{2}$ & $\begin{array}{c}k \times 10^{4} \\
{\left[\mathrm{~s}^{-1}\right]}\end{array}$ & $\begin{array}{c}k \times 10^{4} \\
{\left[\mathrm{~s}^{-1}\right]}\end{array}$ & $\begin{array}{c}k \times 10^{4} \\
{\left[\mathrm{~s}^{-1}\right]}\end{array}$ \\
\hline 2.99 & 4921 & 6262 & 6.5 & 0.971 & - & - & - \\
\hline 3.22 & 39536 & 4416 & 7.74 & 0.990 & 5.23 & - & - \\
\hline 3.31 & 52768 & 3305 & 12.42 & 0.986 & - & - & - \\
\hline 3.40 & 74609 & 2700 & 19.94 & 0.988 & - & - & - \\
\hline 3.48 & 104624 & 1380 & 20.5 & 0.951 & - & 9.05 & - \\
\hline 3.55 & 110987 & 1403 & 23.4 & 0.984 & 35.9 & 12.77 & 15.5 \\
\hline 3.69 & 149132 & 1462 & 20.79 & 0.986 & 31.87 & 14.38 & \multicolumn{2}{|c||}{} \\
\hline
\end{tabular}

Fitting the experimental data to eq. (6), coefficient $k$ is obtained for all 4 cases mentioned above, Table 1. As it is logical to expect, the higher the supersaturation the more probable is the molecule attachment to the sub-critical precursors, i.e. higher $k$ value is obtained. It is worth noting however that all $k$-values for bulk insulin crystal nucleation calculated by means of eq. (8) and using experimental points in the vicinity of function's midpoint for direct determination of $n_{\mathrm{s}}, t_{\mathrm{o}}$ and $t_{\mathrm{s}}$ are systematically lower ( $\leq$ $15 \%$ ) than the $k$-values presented in Table 1. Evidently, the reason is the approximation used in deriving eq. (8), which neglects the starting and final non-steady state periods. However, in view of the tremendous $k$-value divergences with different systems (see §5), this deviation is tolerable.

\subsection{Induction time determination}

The S-shape of the $n$ vs. $t$ dependences brings about the issue of precise determination of the nucleation induction time, $\tau$. It is inversely proportional to the square of supersaturation [25], and therefore is clearly detectable at relatively low supersaturations only. For instance, $\tau \approx 30 \mathrm{~min}$ is found in our experiments with $4 \mathrm{mg} / \mathrm{ml}$ insulin concentration, which renders dimensionless supersaturation $\Delta \mu / \mathrm{k}_{\mathrm{B}} \mathrm{T}=2.99$ (Fig. $3 a)$, while at the higher supersaturations, $\tau$ is hardly noticeable. Since experimental 
determination of $\tau$ is uncertain at high supersaturations, the logistic functional interpretation of $n$ vs. $t$ dependences could help in this respect. Kashchiev [26] shows that

$$
\tau=\left(6 / \pi^{2}\right) t_{\mathrm{o}} \approx 0.6 t_{\mathrm{o}}
$$

Keeping in mind that due to the inherent data scatter, all nucleation rates determined directly from the experimental points depend on the choice of such points, and that the steady-state nucleation rate was best defined by the tangential to the sigmoid's midpoint, which is the maximum of the dimensionless nucleation rate, $(\mathrm{d} n / \mathrm{d} t)_{\max }$, eq. (2), $\tau \approx 32$ min was calculated with $t_{\mathrm{o}} \approx 53$ min obtained from eq. (5), and using $t_{\mathrm{p}}$ and $k$ data from Table 1 .

\section{Comparison with a two-step crystal nucleation process}

In an elegant study of bovine $\beta$-lactoglobulin crystallization, using small-angle $\mathrm{X}$ ray scattering and optical microscopy, Sauter et al. [27] have presented convincing evidence for a two-step crystal nucleation mechanism. The authors observed that initially, crystal number densities increase with time, achieving steady-state augmentation, and finally reaching a constant (saturation) value. Sauter et al. report a realistic scenario for their direct study, according to which an intermediate phase is formed during the first step of the crystallization process. Then, nucleation of crystals follows within the intermediate phase. The nucleated crystals continue to grow, but due to the low mobility in the surrounding dense intermediate phase their growth is slow. In the next step, the intermediate phase is consumed by the nucleation and slow growth, and with its disappearing, the number of the bovine $\beta$-lactoglobulin crystals becomes nearly constant.

This scenario corresponds to a sigmoid $n$ vs. $t$ dependence, in which the nucleation decelerating factors are gradual exhaustion of the intermediate phase (occurring due to crystal nucleation and growth at the expense of the intermediate phase), and its final disappearance. We found that eq. (6) also fits the $n$ vs. $t$ experimental data reported by Sauter et al. in [27], Fig. 5. In doing so, we established that for bovine $\beta$ lactoglobulin crystallization $k \approx 6 \times 10^{-4}\left[\mathrm{~s}^{-1}\right]$, which matches well the one for insulin crystal nucleation in Table 1. 


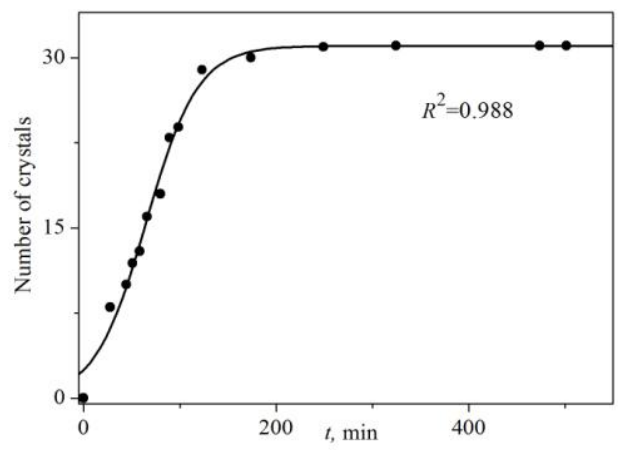

Fig. 5 Logistic function dependence of data for $\beta$-lactoglobulin crystal number, reported by Sauter et al. in [27].

\section{Universality of sigmoid law of new phase nucleation kinetics}

Since nucleation acceleration and deceleration equalize each other over time regardless of the system in which they occur it could be assumed that the logistic (sigmoidal) functional interpretation of nucleation kinetics should be universal. Besides, this interpretation is independent on the specific nucleation scenarios - molecule attachment according to Szilard chain or agglomeration of subcritical clusters (and the implications thereof); direct electron-microscopic observations of colloidal platinum nanocrystal growth exhibit both monomer addition and cluster coalescence events [28] (for diverse fashions of Au-nanorods assembly see Chen et al. [29]).

As it is seen in Table 1, closely similar $k$-values are found for all four cases of insulin crystal nucleation (in bulk solution, on glass surface, at the air/solution interface, and in the three-phase angle air/solution/glass). Approximately the same $k$-value is found for bovine $\beta$-lactoglobulin. Thus, it may turn out that k-value in the same order of magnitude characterize protein crystallization

Unfortunately, however, being interested merely in stationary nucleation rate determinations many experimenters stop there and do not consider the plateau regions. Nevertheless, S-shaped $n$ vs. $t$ curves are published for other systems as well, e.g. for amyloid fibril formation, electrochemical nucleation and glass crystallization. A similar to eq. (6) logistic function has been derived (but under entirely different physical assumptions and using some approximations) and proven experimentally for amyloid fibril formation (that includes both nucleation and growth) by Crespo et al. [3]. In a 
strong contrast to protein crystallization, our logistic-functional plotting of experimental data for electrochemical silver crystal nucleation measured by Milchev [2] renders $k=$ $9130\left[\mathrm{~s}^{-1}\right]$; and for electro-chemical mercury droplet nucleation [30] we calculate $k=$ $\left.1384\left[\mathrm{~s}^{-1}\right]\right)$. Both values are seven orders of magnitude larger than the $k$-values for insulin and bovine $\beta$-lactoglobulin. S-shaped $n / n_{\mathrm{s}}$ dependence on $t$ has been found by Fokin et al. [31], who have studied simultaneous nucleation and growth of $\mu$-cordierite crystals on a polished surface of cordierite glass at temperature $850^{\circ} \mathrm{C}$; due to the extremely low viscosity of the glass system, this dependence renders the smallest $k=3.49 \times 10^{-5}\left[\mathrm{~s}^{-1}\right]$.

As far as $k$ depends on the mechanism of molecule supply from the mother phase to the nuclei, it is strongly influenced by system's type. The very small $k$-values calculated for protein crystal nucleation are due to the exceptionally slow kinetics, which is observed with this system, despite the extraordinary high supersaturations that are needed for nucleation initiation. Recently, this fact has been considered with the introduction of the notion for a bond-selection mechanism [23, 32]. However, it should be added that using electrochemical nucleation, e.g. [33], the supersaturation is imposed instantaneously, while in contrast, due to the slower supersaturation establishment in solutions the cluster distribution accommodation proceeds in a more complicated manner (than in the former case).

\section{Conclusion}

Equation (1) for the new phase nucleation rate is derived without any assumption for a specific material and/or system. Coupled with the experimentally confirmed sigmoidal nucleation time dependence for nine different systems (such as protein and electrochemical nucleation, glass crystallization, and amyloid fibril formation), this fact supports the suggestion that the sigmoidal interpretation of the new phase nucleation kinetics is universal. It joined the classical nucleation theory and the modern two-stage crystal nucleation mechanism, and fitted equally well experimental results on stationary and non-stationary nucleation kinetics.

\section{Acknowledgements}

This work is supported by COST Action number CM1402. The authors thank to the National Science Fond at Bulgarian Ministry of Science and Education for the financial 
support by NSF project No T02/8/12.12.2014. Some discussion with Isak Avramov and Alexander Milchev are acknowledged.

\section{References}

[1] C.N. Nanev, F.V. Hodzhaoglu, I. L. Dimitrov, Cryst. Growth \& Design 11 (2011) 196-202.

[2] A. Milchev, DSc Thesis (Bulgarian), Sofia, Bulgaria, 1998.

[3] R. Crespo, F.A. Rocha, A.M. Damas, P.M. Martins, J. Biolog. Chem. 287 (2012) 30585-30594.

[4] I. Avramov, J. Šesták, J. Thermal Analysis and Calorimetry 118 (2014) 1715-1720.

[5] P.F. Verhulst, Correspondance mathe'matique et physique X (1838) 113-121.

[6] P.F. Verhulst, Mem. Acad. Roy. Bruxelles 18 (1844) 1.

[7] A. Kolmogorov, Bull. Acad. Sci., URSS. Math. Nat. 3 (1937) 355-359.

[8] W.A. Johnson, R.F. Mehl, Trans. Am. Inst. Min. (Metall.) Eng. 135 (1939) 416-458.

[9] M. Avrami, J. Chem. Phys. 7 (1939) 1103-1112.

[10] M. Avrami, J. Chem. Phys. 8 (1940) 212-224.

[11] M. Avrami, J. Chem. Phys. 9 (1941) 177-184.

[12] C.N. Nanev, Theory of Nucleation, in: T. Nishinaga (Ed.), Handbook of Crystal Growth, second ed., Vol. I., Elsevier, Amsterdam, 2015, pp. 315-358.

[13] J.L. Robins, T.N. Rhodin, Surf. Sci. 2 (1964) 346-355.

[14] R. Kaischew, B. Mutaftschiew, Electrochim. Acta 10 (1965) 643-650.

[15] D. Kashchiev, Nucleation; basic theory and applications, Butterworth-Heinemann, Oxford, 2003; Chapter 32 and references therein.

[16] A.A. Milchev, Russian J. Electrochem. 44 (2008) 619-664.

[17] G. Tammann, Aggregatzustaende, Die Zustandsaenderungen der Materie in Abhaengigkeit von Druck und Temperatur, second ed., von Leopold Voss, Leipzig, 1923, pp. 222-223.

[18] O. Galkin, P.G. Vekilov, J. Am. Chem. Soc. 122 (2000) 156-163.

[19] N. Candoni, Z. Hammadi, R. Grossier, M. Ildefonso, E. Revalor, N. Ferté, T.

Okutsu, R. Morin, S. Veesler, Int. J. Nanotechnol. 9 (2012) 439-459.

[20] M. Ildefonso, N. Candoni, S. Veesler, Org. Process Res. Dev. 16 (2012) 556-560. 
[21] M Ildefonso, N Candoni, S Veesler, Cryst.Growth \& Des. 13 (2013) 2107-2110.

[22] E. Revalor, Z. Hammadi, J.-P. Astier, R. Grossier, E. Garcia, C. Hoff, K. Furuta, T.

Okustu, R. Morin, S. Veesler, Journal of Crystal Growth, 2010, 312 (7), pp. 939-946.

[23] C.N. Nanev, Prog. Cryst. Growth Charact. Mater. 59 (2013)133-169.

[24] C.N. Nanev, F.V. Hodzhaoglu, Cryst. Res. Technol. 47 (2012) 1195-1200.

[25] S. Toschev, Homogeneous nucleation, in: Crystal Growth: an introduction, P. Hartman (Ed.), North-Holland Publ. Co., Amsterdam, 1973, pp. 1-49.

[26] D. Kashchiev, Surf. Sci. 14 (1969) 209-220.

[27] A. Sauter, F. Roosen-Runge, F. Zhang, G. Lotze, R.M.J. Jacobs, F. Schreiber, J. Am. Chem. Soc. 137 (2015) 1485-1491.

[28] J.M. Yuk, J. Park, P. Ercius, K. Kim, D.J. Hellebusch, M.F. Crommie, J.Y. Lee, A. Zettl, A.P. Alivisatos, Science 336 (2012) 61-64.

[29] Q. Chen, H. Cho, K. Manthiram, M. Yoshida, X. Ye, A.P. Alivisatos, ACS Cent. Sci. 1 (2015) 33-39.

[30] S. Toschev, I. Gutzow, Kristall und Technik 7 (1972) 43-73.

[31] V.M. Fokin, N.S. Yuristin, V.N. Filipovich, A.M. Kalinina, J. Non-cryst. Solids 219 (1997) 37-41.

[32]. C.N. Nanev, J. Cryst. Growth 402 (2014) 195-202.

[33] A.A. Milchev, Electrocrystallization: Fundamentals of Nucleation and Growth, Kluwer Academic Publishers, Boston/Dordrecht/London, 2002. 